\title{
SEAFAC ACOUSTIC SENSORS AND INSTRUMENTATION
}

\author{
R. D. Weiland \\ Naval Surface Warfare Center, Carderock Division \\ Detachment Puget Sound \\ Bremerton, Washington 98314-5215
}

\begin{abstract}
The success of the submarine noise reduction program has dramatically reduced the radiated noise from U.S. submarines. Lower submarine radiated noise levels coupled with increased ocean traffic has created a challenging measurement problem. As submarines have gotten quieter the ocean they operate in has become noisier, thus making it more difficult to measure submarine radiated noise. It is important to make the distinction between a detection system and a measurement system. A detection system can operate at very low signal to noise ratios and still answer the question is there anything there? A measurement must go beyond detection, but must provide answers to additional questions. What is the absolute sound pressure level? What is the source(s)? The area where the distinction between a detection and measurement system is most noticeable is the type and quality of data. A measurement system provides the engineering data necessary to affect changes in submarine design and/or submarine systems design. The accuracy and repeatability of the measurement is central when considering that data from the measurement will be utilized in deciding whether a multi-million dollar ship alteration or design modification is pursued. Among the most important criteria in the design and implementation of a measurement system for submarine radiated noise are: an understanding of the submarine noise spectra, measurement site environment, acoustic sensor selection and instrumentation. The design of the SEAFAC measurement system will be viewed as a sequential consideration of these criteria.
\end{abstract}

\section{INTRODUCTION}

The Southeast Alaska Acoustic Measurement Facility (SEAFAC) is the Navy's newest acoustic measurement facility. The recently completed measurement facility provides an integrated submarine tracking, measurement, signal processing and data analysis facility. The SEAFAC measurement site was selected from a host of other potential sites after years of environmental data collection. Behm Canal, located in the Alaskan panhandle near Ketchikan was found to provide the best acoustics of all the sites considered.
Behm Canal was formed by a glacial cut and has a nominal depth of 1,200 feet. The bottom is characteristic of glaciated fjords. It is soft, and composed of glacial till material that has a porosity of 0.8 . The ambient noise for Behm Canal can be characterized as that from a sheltered body of water and exhibits normally quiet acoustic conditions. The 20 percentile curve is less than or equal to Knudsen SS $1 / 2$, the 50 percentile curve is nominally Knudsen SS 1, and the 80 percentile curve falls between Knudsen SS2 - SS3 or quieter [1].

\section{A. Submarine Noise Source Characteristics and Measure- ment System Considerations}

The site selection and measurement system design process must consider what is to be measured and the environment where it is to be measured. The radiated acoustic energy measured from modern submarines can be characterized as having components of machinery noise, propeller noise and hydrodynamic flow noise [2]. The sound pressure level, frequency distribution and dominant noise contributor to the submarine's signature varies with the submarine's operational configuration. When operating at high speeds the submarine's radiated signature is dominated by the noise contributions of the propeller and propulsion related machinery [3] [4]. While operating at the lower speeds, the machinery components are known to dominate the radiated signature.

A study of the amplitude and frequency distribution of the range site's ambient noise and the submarine's radiated noise signature under various operating conditions will provide a basis for answering several important site selection and range design questions. Is the range site quiet enough to make the measurements? What are the requirements for acoustic sensors? Will omnidirectional hydrophones be sufficient or will array gain be required? What dynamic range is required? What is the lowest sound pressure level required to be measured? What are the noise floor requirements? What is the highest sound pressure level to be measured? 


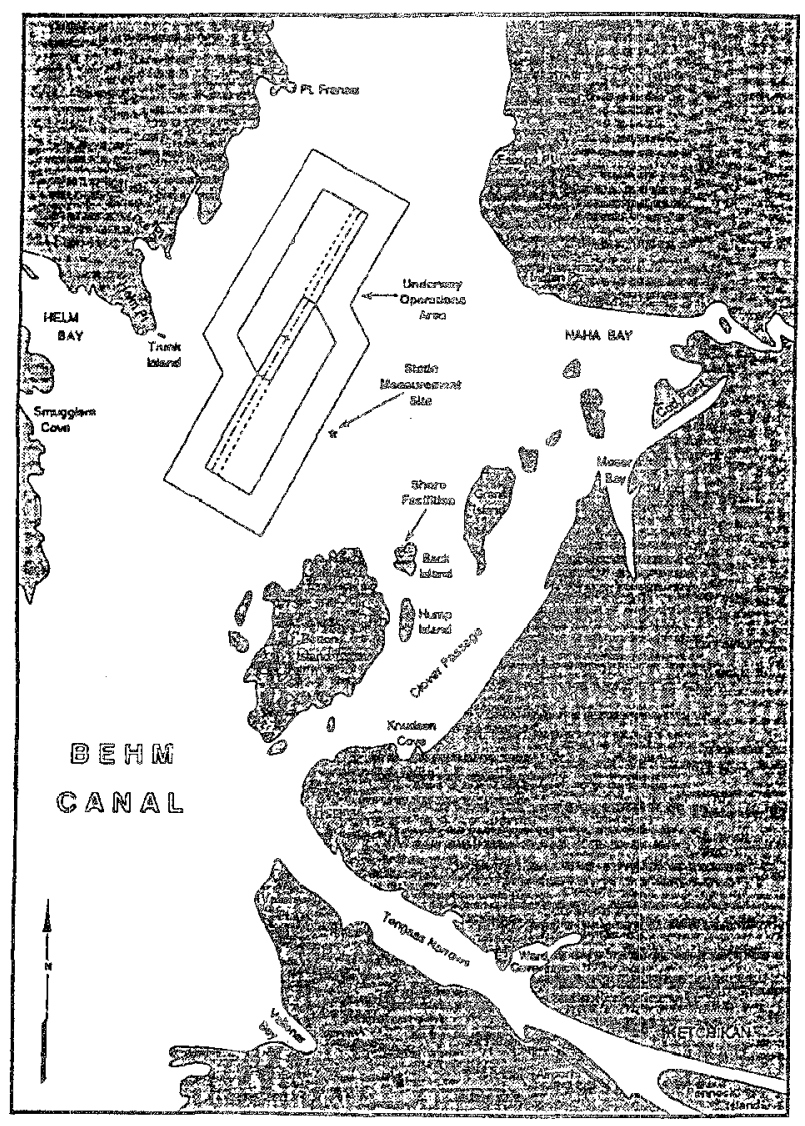

Fig. 1. SEAFAC range arsa.

\section{SEAFAC RANGL CONEIGURATION}

The range area shown in Fig. (1) is divided into two operational sites, an underway sitc and a static site. The underway site is where the submarine maneuyers between two measurement arrays at various speeds, depths, and machinery conditions. The radiated energy from a transiting submarine is received by two vertical arrays of hydrophones spaced 250 yards apart. The submarine transits between the two arrays, allowing simultarneous measurement of port and starboard radiated noise signatures. The arrays are installed such that the vertical center of the two arrays are within 10 feet of the same depth. The depth offset requirement ensures that the submarine will be operating within the main response lobe of the directional line array hydrophones. The underway operations area is characterized by a 5,000 by 12,000 yard rectangle. The static site suspends the submarine by cables attached to flotation barges. While suspended the propulsion system and engineering plant are secured eliminating their noise contribution from the signature. This allows the remaining machinery to be cycled and its contribution to the total acoustic signature to be evaluated.
While operating at SEAFAC the submarine's position is continuously monitored as it maneuvers through the measurement area with the aid of an acoustic tracking and navigation system. The submarine is outfitted with a dual frequency, (16 kHz \& $75 \mathrm{kHz})$ phase shift keyed pinger which is tracked throughout the operational area. The pinger's frequency, repetition rate, and power output, is controlled by the operating submarine. The computed position is sent to the operating submarine via an acoustic data link where it is decoded and displayed onboard.

\section{ACOUSTIC MEASUREMENT ARRAYS}

Cost and risk were the driving concerns in the development of the acoustic measurement arrays. To minimize both, the approach was to make use of existing sensor and array technology. Each hydrophone array system can be described as a bottom moored, taut line suspended from subsurface buoys. The "taut line" is a multiconductor signal cable with an integral synthetic fiber strength member. This cable is referred to as the riser cable and attached to it are six omnidirectional hydrophones, two directive line arrays, and an in-line electronics bottle. Fig. (2) illustrates the array configuration. The acoustic sensors are attached to the array via stand offs manufactured from plastic (PVC) sheet stock. The omnihydrophones are placed at depths of $200,300,400,500,600$, and 800 feet. The crystal elements in the omnihydrophones are identical to the phones currently in use at the Carr Inlet Acoustic Range (CIAR) and at the Santa Cruz Acoustic Range Facility (SCARF). The hydrophones are useable from $5 \mathrm{~Hz}$ through $100 \mathrm{kHz}$. The directive arrays provide array gain in the mid and highfrequencies bands. The Mid-frequency Directive Array (MDA) is a nested aperture vertical line array. The MDA consists of a 31 foot oil filled hose and an outboard electronics Pressure Vessel (PV). The hose contains 17 hydrophone elements which are routed to the PV. Inside the PV an integral time delay beamformer forms three nested apertures with design frequencies of $800,1,600$, and $3,200 \mathrm{~Hz}$. The hydrophone element shading is controlled from the Range Operations laboratory. The available weights are uniform, binomial, Taylor or Sin $x / x$. The Highfrequency Directive

Array (HDA) has an overall length of 84 inches. The acoustic sensing portion is a 63 inch long stack of crystals with signal taps at the $1,2,4,8,16$, and 32 inch points. This provides six directional hydrophone elements with different apertures. The HDA exhibits directional characteristics in the vertical (X-Z) plane; and is omnidirectional in the horizontal (X-Y) plane. The effective operating frequency of the array spans a range from $500 \mathrm{~Hz}$ to $80,000 \mathrm{~Hz}$. The SEAFAC implementation uses only the 4, 


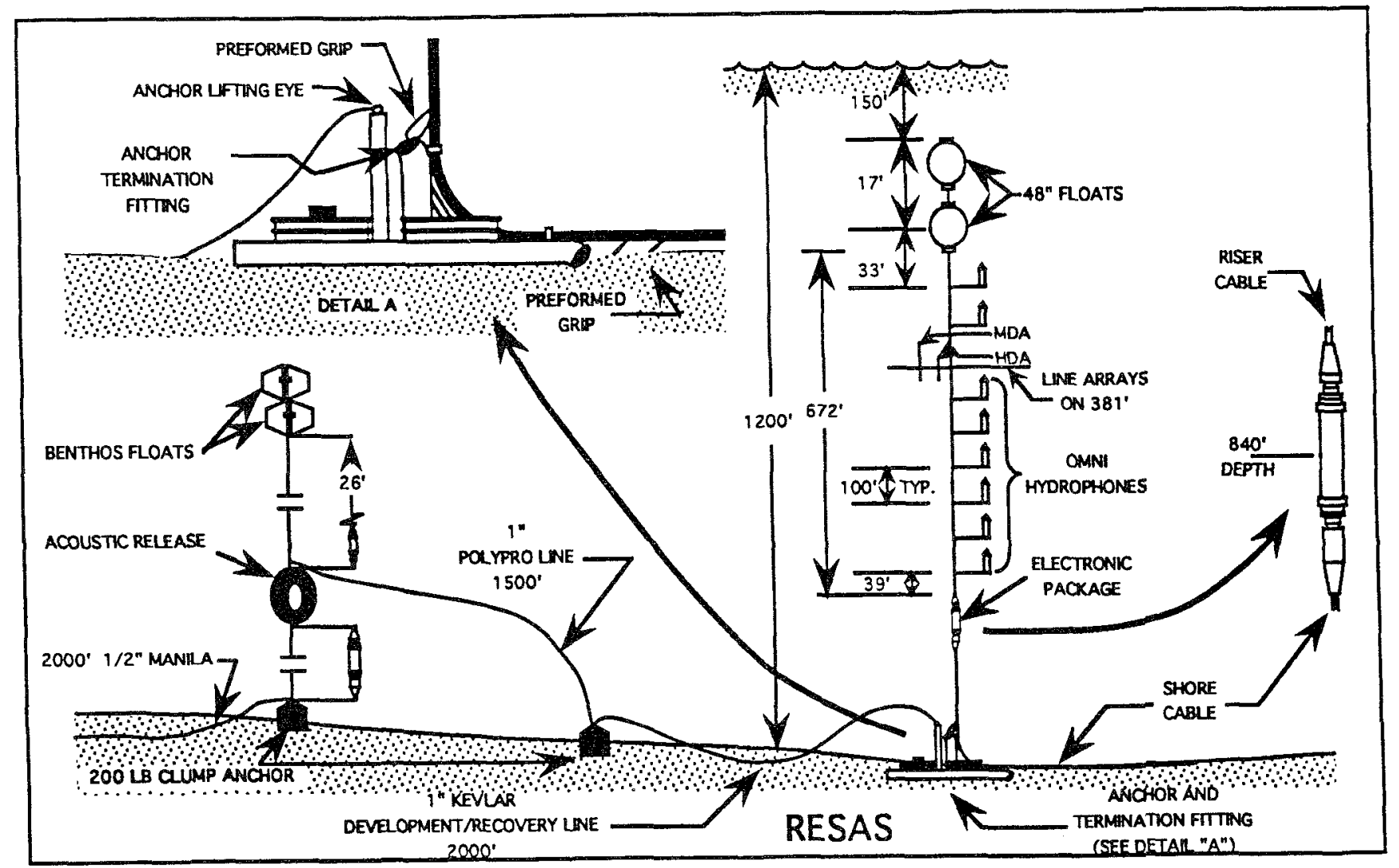

Fig. 2. Hydrophone array configuration.

8,16 , and 32 inch elements. Each directive array is attached such that the Maximum Response Axis (MRA) is located at $381 \mathrm{ft}$. An electronics bottle houses the line drivers and voltage regulators for the individual hydrophones and array systems. In addition, a pseudo-random noise source for conducting electrical calibration is included in the bottle electronics.

\section{DATA TRANSMISSION}

The acoustic energy received by the arrays is transmitted to the shore complex for processing. The arrays are connected to the shore instrumentation through an armored, shielded 12-quad signal cable. The hydrophone preamps produce a true differential output signal. The hydrophone signals are routed to the electronics bottle where they are amplified and impedance matched to drive the signal over the approximately 28,000 feet of cable from electronics bottle to shore. To obtain adequate signal levels at the shore end of the cable the low level hydrophone outputs required additional gain to overcome the cable losses. The electronics bottle contains additional gain stages which are designed to compensate for the loss in the cable. Field measure- ments show that signal levels measured at the hydrophone outputs are within $2 \mathrm{db}$ of the levels measured at the shore end of the cable. A shielded quad was allocated to each omni hydrophone. Two of the four wires within the quad are allocated to DC power, while the second pair is dedicated to hydrophone signal output. The line array signals are transmitted in a similar

manner.

\section{A. Cable Installation}

The signal cable routes were selected with future retrieval in mind, for maintenance and/or repair. The cable routes were laid out such that the paths of the array cables would not interfere with other cables. The 12-quad cables were laid with a 300 foot separation shoreward from the array implant position. Fig. (3) shows the cable routes. Neither of the two 12-quad cables cross over another cable or each other. During the summer of 1991, 14 individual cables were installed by MARIPRO using the R/V McGaw. Each cable lay was approximately 30,000 feet. As part of the installation design the near shore cable landing areas were investigated by divers. A site was selected where PVC conduits could be installed from the shore out. The 


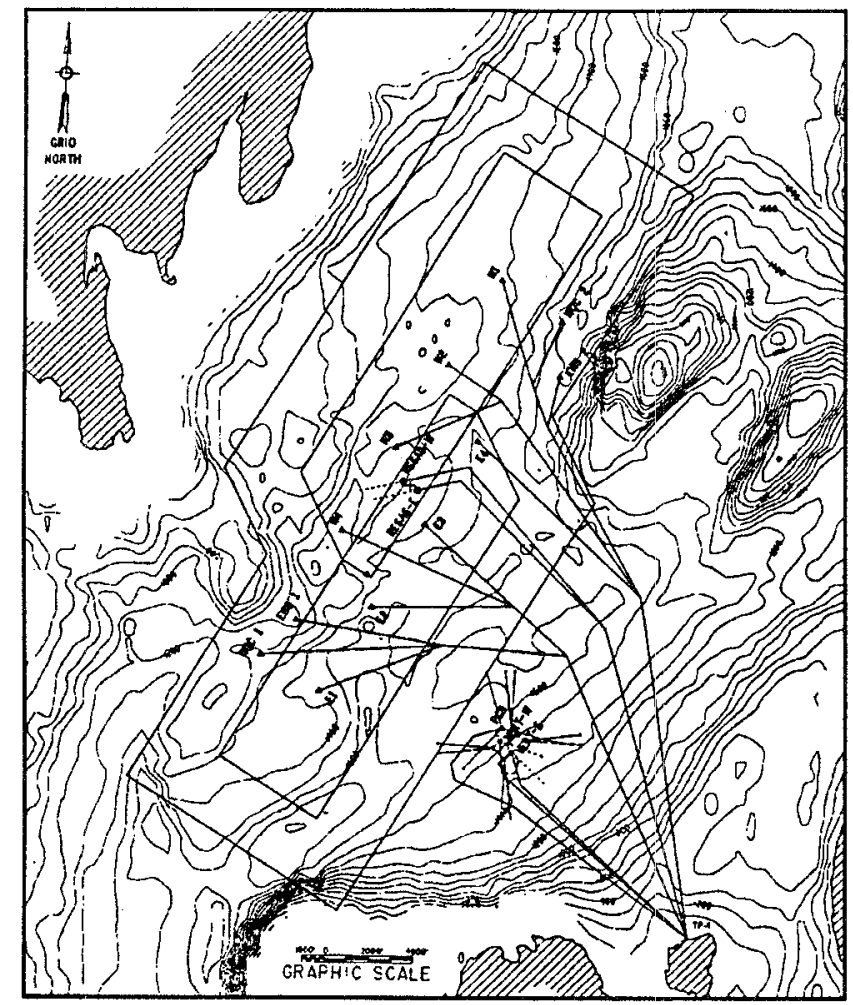

Fig. 3. Underwater cable routes.

conduits were installed out to a depth of minus 10 feet below MLLW. The conduits were installed in a cable trench and covered with a six inch layer of concrete. One conduit was provided for each cable to be installed. Spare conduits were installed for the static site arrays and for future expansion. The hydrophone array was installed first, then the 12-quad cable was laid from the array to the shore. At the shore landing area, the cable was pulled through a conduit into the cable trench to the range operations building. The hydrophone array cables are terminated at a transition cabinet that is mounted in the cable chaseway on the front of the Range Operations building. The armored cables enter the transition cabinet through water tight stuffing tubes. The individual quad cables are connected to terminal blocks in the transition cabinet from which twisted, shielded pair cables route to a termination cabinet located in the operations laboratory.

\section{LABORATORY INSTRUMENTATION}

The function of the laboratory instrumentation is to terminate the array cables, provide DC power, provide a control interface for adjusting the array shading, amplification, signal conditioning, distribution, recording and real time monitoring of the array hydrophone signals. When terminating a long cable system it is common practice to establish a low impedance ground and terminate the cable shields to it [5] [6]. Terminating the shore cable to the earth reference can establish a current flow. These currents are a result of the cable shield simulating an antenna and receiving both man made and naturally occurring electro-magnetic signals. Inevitably, even instrumentation amplifiers with good common mode characteristics "pick up" these currents as "noise" residing on the shields. The proximity of the shore amplifiers to this high current noise source, the shield tie point to the earth reference is often overlooked. If the shields were not tied to earth reference, there would be no current flow. In contrast, the better the connection to earth reference, (lower impedance) the greater the current flow. The grounding scheme used in the SEAFAC array system is completely isolated from the earth reference. The use of differential amplifiers in the hydrophone preamps, line drivers and shore amplifiers has enabled the establishment of a local, zero signal reference through out the system. Powerline frequency noise levels have been measured at $-156 \mathrm{db}$ re 1 volt in the acoustics laboratory. The termination cabinet contains the zero signal reference plane for the shore instrumentation, power supply decoupling circuits and interface to the balanced input, low noise variable gain, amplifiers. Power for the hydrophone arrays is provided by DC power supplies located in the operations laboratory. The hydrophone array power supplies are configured to operate in the current limit mode, providing constant current to the electronics bottle regulators. The post amplifiers provide signal line impedance matching, common mode rejection, signal equalization, amplification and filtering. The outputs of the amplifiers are routed to monitor oscillopes, from there the signals are connected to signal distribution panels, where the signals are buffered and routed to two 28 track analog tape recorders, signal monitoring equipment, and to the signal processing system. The signal monitoring equipment suite provides "real time" monitoring of the incoming signals from the hydrophone arrays. The monitoring capabilities include aural, narrowband, and one third octave band displays, Low Frequency Analyzing Recorder (LOFAR) gram, level versus time, and filtered level versus time. The "real time" monitoring equipment suite is independent of the signal processing equipment. This allows the user to manipulate and examine the real time signals and not be concerned about impacting the data collection.

\section{DATA COLLECTION CAPABILITIES}

The signal processing system utilizes a distributed architecture for robustness and ease of development. The system is segmented along two independent halves, a data acquisition half and a data analysis half. The data acquisition segment collects 52 simultaneous acoustic channels. Twelve channels are dedicated to one-third octave (OTO) 
processing, spanning bands 8 to 52 . OTO is accomplished using six dual channel Larson-Davis 3100 analyzers. The remaining 40 acoustic channels are digitized and processed using a 8192 point Fast Fourier Transform (FFT). The sample rates are adjusted based on the upper frequency selected for the channel. The FFT processing provides 3,200 useable frequency bins per channel. Of the 40 narrowband channels up to 16 can be assigned to LOFAR processing which produces LOFAR "grams" on two CRT's.

\section{SUMMARY}

The SEAFAC implementation of sensors and instrumentation offers the users an integrated acoustic test and measurement facility. The sensor and instrumentation suite provides the capabilities required for conducting a full spectrum of acoustic measurements.

\section{ACKNOWLEDGMENTS}

The following organizations are acknowledged for their contributions to the SEAFAC project: The Naval Sea Systems Command, PMS390; the Naval Facilities Command, Chesapeake Division (NAVFAC CHESDIV); MARIPRO a division of Science Applications International Corp; Applied Measurement Systems, Inc.; and West Sound Associates. The author wishes to acknowledge separately the contributions of Norman Miller, West Sound Associates who's guidance, insight, and support whose have been instrumental in the successful development of this facility.

\section{REFERENCES}

[1] J. Elterich, M. Schilt "SEAFAC Underwater Ambient Noise", Ship Acoustics Department, Dec 1991

[2] D. Ross, " Mechanics of Underwater Noise", Pergamon Press Inc., 1976, pp. 253-345

[3] R. Urick, "Principles of Underwater Sound",3rd Ed, McGraw-Hill, 1983, pp. 328-403

[4] R. Coates, "Underwater Acoustic Systems",John Wiley \& Sons, 1989 [5] R. Morrison, "Grounding \& Shielding Techniques in Instrumentation", 2nd Ed, John Wiley \& Sons, 1977

[6] H. Ott, "Noise Reduction Techniques in Electronic Systems", 2nd Ed, John Wiley \& Sons, 1988 